\title{
Vector Boson Scattering prospects for High-Luminosity LHC at CMS in the same sign WW final state
}

Raffaele Gerosa*t

INFN and University of Milano Bicocca

E-mail: Raffaele.Gerosa@mib.infn.it

A feasibility study is presented for Vector Boson Scattering (VBS) measurements in the samesign WW final state in view of the high luminosity phase of the LHC (HL-LHC). Particular emphasis is given to the expected performances of the CMS upgraded detector, which is compared to the results attained with the current aged and non-aged technology. Expected sensitivity to the longitudinal electroweak scattering, the non-unitarized scenario, in which the Higgs boson does not regularize the scattering cross section, and to anomalies in the quartic gauge couplings have been set through dedicated simulation studies.

XXVII International Symposium on Lepton Photon Interactions at High Energies

17-22 August 2015

Ljubljana, Slovenia

* Speaker.

${ }^{\dagger}$ On behalf of the CMS Collaboration 
Precision measurements in the electroweak symmetry breaking (EWSB) sector represent important goals in the context of the high luminosity LHC program. Vector Boson Scattering (VBS) and quartic gauge couplings (QGC) are key aspects of the Standard Model (SM), which remain mainly unexplored after the first run of the LHC collisions. In fact, so far only an evidence for the electroweak WW production has been reported by both ATLAS and CMS experiments [1,2]. The observed Higgs boson provides a candidate for a simplified EWSB mechanism, where vector bosons acquire mass and a longitudinal degree of freedom through their couplings to the Higgs field. These interactions allow also the unitarization of the scattering amplitudes of longitudinally polarized vector bosons $\left(\mathrm{V}_{\mathrm{L}} \mathrm{V}_{\mathrm{L}} \rightarrow \mathrm{V}_{\mathrm{L}} \mathrm{V}_{\mathrm{L}}\right)$, preventing the final result to be divergent at high energies [3]. If the SM is just an effective low energy theory, the VBS unitarization will be only partially operated by the Higgs boson and the complete regularization would happen via new physics, which intervenes at a large energy scale. In a large data sample, VBS may be used to determine that scale and possibly resolve different models that could be nearly degenerate at the Higgs pole, while showing different behaviors at higher energies.

This poster presents studies of the CMS experiment potential at the HL-LHC inside this context [4]. The VBS signal exhibits when two vector bosons, emitted by a pair of incoming quarks, interact with each other. The two quarks get deflected from the beam direction and originate strong hadronic jets in the detector, called tag-jets, which clearly identify a scattering event. In this way, the HLLHC becomes a vector boson collider, providing access to purely electroweak processes, $\mathrm{O}\left(\alpha_{e w}^{6}\right)$ at Leading Order (LO), in a high energy regime. Any new resonances in the VBS invariant mass spectrum or a deviation of data from the SM expectations would be a ground-breaking result, a direct evidence of the new physics scale. The small cross section of the electroweak process, $\mathrm{O}\left(\alpha_{e w}^{6}\right)$, and the large contamination due to the production of vector boson pairs in association with two hadronic jets via strong interactions, $\mathrm{O}\left(\alpha_{e w}^{4} \alpha_{s}^{2}\right)$, make this analysis very challenging.

Moreover, in the harsh experimental conditions of the HL-LHC, an average of about $140 \mathrm{soft}$ QCD interactions (pileup) will overlap with each other during the same beam collision. In this high-occupancy environment, background contamination also arises when hadronic jets are misidentified as leptons ( $j$ - $\ell$ fake rate), or jets from pileup interactions mimic the properties of VBS ones. For these reasons, the designed upgrade of the CMS detector (Phase-II) presents a new tracking system, extended up to $|\eta|=4$, and a new radiation hard and highly granular forward calorimeter, which are beneficial in reducing the $j-\ell$ fake rate as well as the background produced by pileup events. In this study, only fully leptonic decays of the vector bosons are considered, to select topologies with lower backgrounds at hadron colliders, avoiding ambiguities due to the separation of the tag jets from the vector boson decay products. Projections are presented for same-sign WW scattering $\left(p p \rightarrow W^{ \pm} W^{ \pm} j j \rightarrow 2 \ell^{ \pm} 2 v j j\right)$.

The sensitivity to the same-sign WW electroweak scattering is determined through the measurement of its inclusive and longitudinal cross sections. The sensitivity to a non-unitarized scenario, in which the Higgs boson does not play any role in the VBS, is studied as an indicator for the determination of new strongly-coupled sectors in the theory. Finally, new physics resulting in enhanced VBS cross section at high energy could be due to high-mass scalar resonances or new massive vector bosons, such as those predicted in unified theories. Such additional states would alter the strong interference between the VBS diagrams, leading to strong enhancements at high energy. These effects are described through the effective field theory (EFT) formalism, where dimension-six op- 
erators are well constrained by other measurements, while VBS is modified by dimension-eight operators through anomalous QGC (aQGC) [5]. These benchmarks are tested for different CMS detector configurations. VBS signal and irreducible backgrounds (WW $\mathrm{O}\left(\alpha_{e w}^{4} \alpha_{s}^{2}\right), \mathrm{WZ} \mathrm{O}\left(\alpha_{e w}^{6}\right)$ and $\left.\mathrm{O}\left(\alpha_{e w}^{4} \alpha_{s}^{2}\right)\right)$ have been generated at LO via both Phant om [6] and MadGraph [7] generators, while the detector response has been simulated parametrically via Delphes [8]. The aging effect on the CMS detector is determined in the detailed simulations and parametrized according to its effect on selection efficiencies and systematics uncertainties on the physics objects. The $j$ - $\ell$ fake rate is measured with the detailed simulation of the CMS experiment accounting for the specific conditions of each detector scenario. Anomalous couplings are studied in the frame of the EFT formalism [9] implemented in MadGraph, while the non-unitarized scenario is simulated via Phantom.

Selections have been optimized to collect, with the best efficiency, fully leptonic same-sign WW scattering events as well as to reduce background contaminations. First, two same-sign isolated leptons (e or $\mu$ ), with $p_{T}^{\ell}>20 \mathrm{GeV}$ and $\left|\eta_{\ell}\right|<2.4$, are required together with at least two jets (anti-k $\mathrm{k}_{t}$ $R=0.4$ ), with $p_{T}^{j}>30 \mathrm{GeV}$ and $\left|\eta_{j}\right|<4.7$, well separated from the identified leptons $\left(\Delta R_{j \ell}>0.3\right)$. Additional loosely identified leptons in the event are vetoed to reduce the $p p \rightarrow W Z j j \rightarrow 3 \ell v j j$ contribution. This background, together with contaminations from $Z / \gamma^{*}+$ jets events, is further reduced requiring, in the same flavor final state, the di-lepton invariant mass $\left(m_{\ell \ell}\right)$ to be far from the Z-boson mass peak $\left(m_{Z} \pm 10 \mathrm{GeV}\right)$. Moreover, to limit contributions from $\mathrm{W}+$ jets and multi-jet events, which can be wrongly selected when jets are mis-identified as leptons, both $m_{\ell \ell}$ and the missing transverse energy $\left(E_{T}^{\text {miss }}\right)$ must be larger than $40 \mathrm{GeV}$. A sizable reduction of same-sign WW QCD background $\mathrm{O}\left(\alpha_{e w}^{4} \alpha_{s}^{2}\right)$ is achieved requiring the $\eta$-distance between the two leptons $\left(\Delta \eta_{\ell \ell}\right)$ to be less than two units. Semi-leptonic top pairs production $\left(t \bar{t} \rightarrow W^{ \pm} b W^{\mp} \bar{b} \rightarrow \ell v j j b \bar{b}\right)$ reprensents the main source background when one of the jets, either from the hadronic W-boson decay or from a b-quark or any additional hard jet in the event, is wrongly identified as a charged lepton. To reject these events, no jet with $p_{T}>30 \mathrm{GeV}$ should be identified as coming from a bquark by the CMS CSV algorithm [10], then events are discarded if an additional soft muon, with $p_{T}>5 \mathrm{GeV}$, is matched to a reconstructed jet. Since the signal is a pure electroweak process, little color flow is expected in the event. Therefore, events are required to have $\mathrm{H}_{\mathrm{T}}<125 \mathrm{GeV}(150 \mathrm{GeV}$ if the tracker extends up to $|\eta|=4$ ), where $\mathrm{H}_{\mathrm{T}}$ is the energy flow measured with jets composed only by tracks associated to the leading vertex. This quantity is insensitive to pileup and shows a good rejection power against $t \bar{t}, \mathrm{~W}+$ jets and same-sign $\mathrm{WW} \mathrm{O}\left(\alpha_{e w}^{4} \alpha_{s}^{2}\right)$. The two highest $p_{T}$ jets in the event are selected as VBS tag-jets, their pseudorapidity separation $\left(\Delta \eta_{j j}\right)$ is required to be larger than 2.5, while the tag-jet system invariant mass $\left(m_{j j}\right)$ must be larger than $850 \mathrm{GeV}$.

A wide set of observables has been investigated for each of the benchmarks, including the kinematic properties of single objects and their angular correlations. The analysis performance is determined with a two-dimensional binned likelihood fit based on templates of the most sensitive observables. The selected events are classified in eight categories according to the charge and flavour of the final state leptons. Figure 1 (left) shows the $\Delta \varphi$ between the two selected leptons, resulting from the event selection previously described, and considering the CMS upgraded detector (Phase-II). Yields are normalized to $3 a b^{-1}$. The electroweak scattering is represented by the solid blue line, stacked over the expected backgrounds. The dashed purple line shows the different expectation in the extreme case when the Higgs boson does not play any role in the scattering unitarization (noHiggs scenario). The difference between the Higgsless scenario and the SM one is displayed with 
a solid red line. Moreover, Figure 1 (middle) shows the predicted shapes for the different polarized scattering components and the total background for the leading lepton $p_{T}$ distribution, which represents one of the most sensitive observables for measuring the longitudinal cross section.

Finally, in the right plot, the $m_{l l}$ distribution is shown after all selection requirements are applied, showing the expectation for the SM case and for a signal hypothesis with enhanced anomalous couplings.
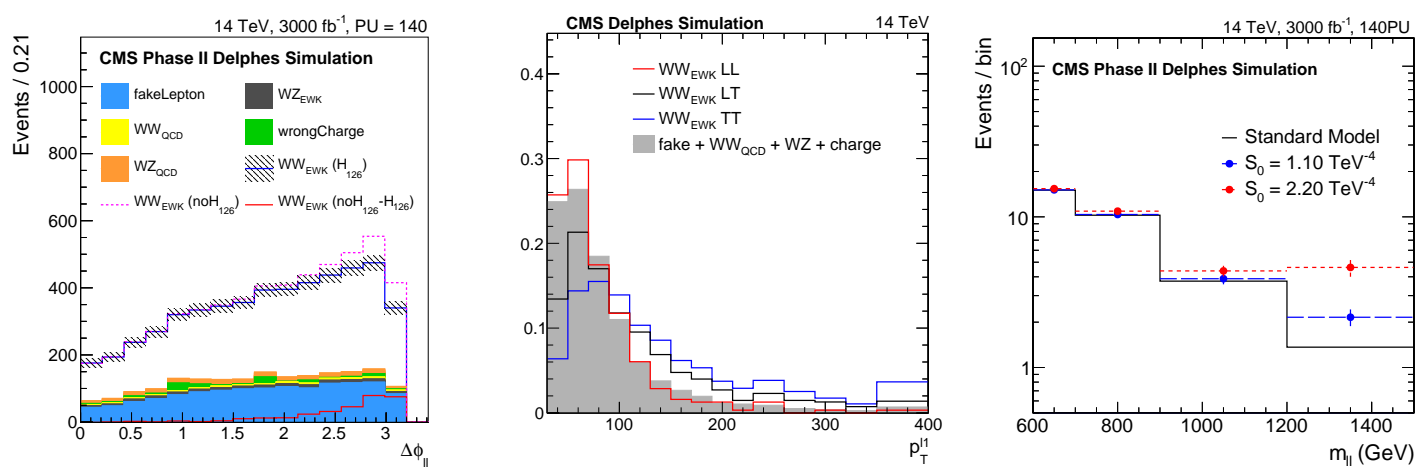

Figure 1: Left, the $\Delta \varphi_{\ell \ell}$ distribution obtained from the two selected final state charged leptons considering the Phase-II detector scenario, with an average of 140 pileup events per bunch crossing. Middle, the leading lepton $p_{T}$ shapes expected for the total background and the electroweak scattering signal split in the exclusive polarization states of the final state W-bosons. Right, the $m_{\ell \ell}$ distribution showing the expectation for the SM case and for a signal hypothesis with enhanced anomalous couplings through the $S_{0}$ operator.

Applying the designed event selection strategy, the inclusive electroweak cross section of samesign W-bosons $\mathrm{O}\left(\alpha_{e w}^{6}\right)$ will be determined, after $3 a b^{-1}$ of integrated luminosity, with a precision at the order of $5 \%$ accounting for the main expected sources of uncertainty. Figure 2 shows the expected significance for the discovery of the longitudinal same-sign WW scattering, obtained from a two-dimensional fit to the $\left(\Delta \varphi_{j j}, p_{T}^{\ell_{1}}\right)$ variables, as a function of a scale factor applied to the jetto-lepton fake rate (left) and the total integrated luminosity of the data sample (right). A discovery sensitivity of about $2.5 \sigma$ is expected for the CMS upgraded detector after $3 a b^{-1}$ and for a unity fake rate scale factor. Figure 3 shows the expected 95\% CL exclusion limit for the Higgsless scenario, obtained from a two-dimensional template fit to the $\left(\mathrm{R}, m_{\ell \ell}\right)$ variables, where $\mathrm{R}$ is defined as in [11]. The result is reported in terms of the strength modifier $(\mu)$ of the difference compared to the SM case. Since the exclusion sensitivity is reached after about $150 \mathrm{fb}^{-1}$, when higher integrated luminosities will be reached, the analysis will become sensitive to partially unitarized scenarios, where the Higgs boson does not fulfill alone to the VBS unitarization. Finally, Figure 4 (left) shows the expected 95\% CL limits on the coefficients for the BSM higher-order operators describing aQGC in the effective Lagrangian. Since the deviations from the SM happen in high energy tails of the distributions and the results approach the systematic limit after $3 a b^{-1}$ of data, the difference between the aged and the upgraded scenario is smaller than the other benchmarks, typically at the order of $10 \%$.

In conclusion, these feasibility studies show how the upgraded detector will recover the performances of the current one in the harsh HL-LHC environment, where the performance improvements with respect to the aged scenario will be necessary for the EWSB verification in a modelindependent way with respect to the detailed description of the SM Higgs resonance. 

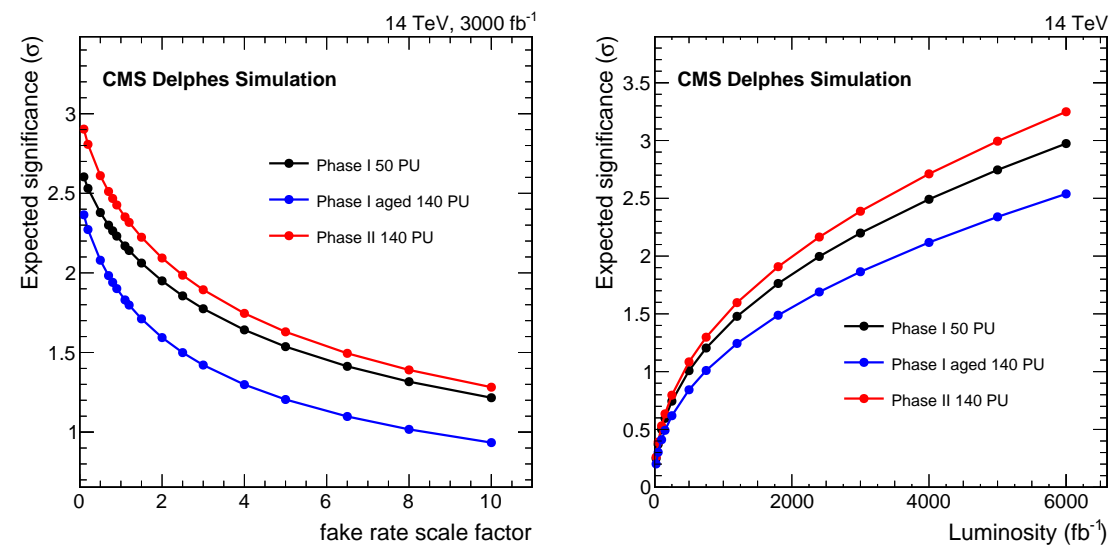

Figure 2: The expected significance for the longitudinal same-sign WW scattering discovery: (left) after $3 a b^{-1}$ of integrated luminosity as a function of the jet-to-lepton fake rate scale factor, (right) as a function of the integrated luminosity for a unity fake rate scale factor.
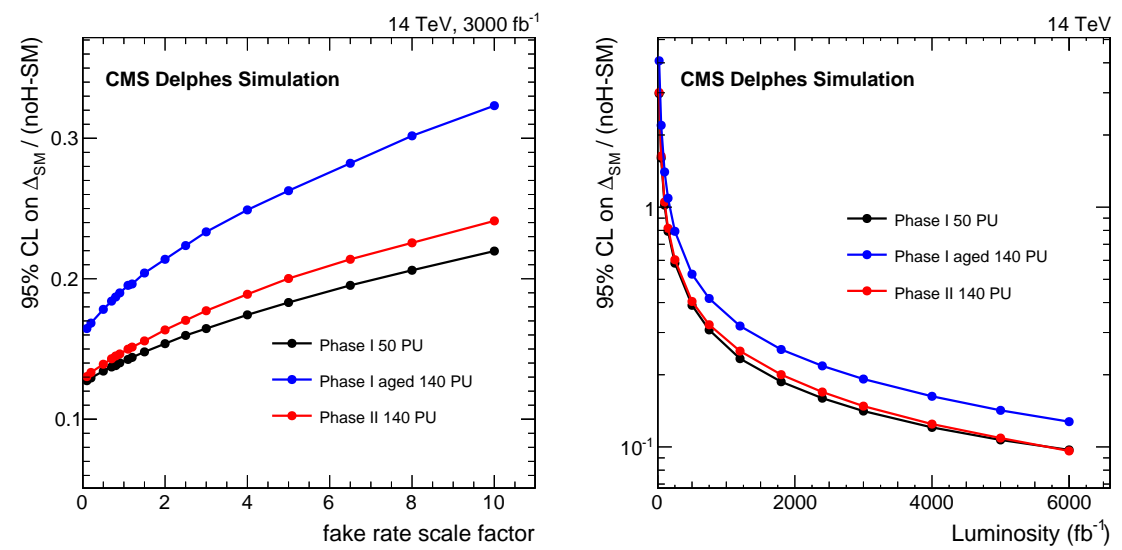

Figure 3: The 95\% CL exclusion limits for the Higgsless scenario: (left) after $3 a b^{-1}$ as a function of a jet-to-lepton fake rate scale factor, (right) as a function of the integrated luminosity for a unity scale factor. Limits are expressed as deviation from the SM divided by the difference of the Higgsless case from the SM.

\begin{tabular}{|l|c|c|c|}
\hline & Phase-I & Phase-II & Phase-I aged \\
\hline $\mathrm{S}_{0}$ & 1.06 & 1.07 & 1.17 \\
$\mathrm{~S}_{1}$ & 3.51 & 3.55 & 3.87 \\
$\mathrm{M}_{0}$ & 0.78 & 0.75 & 0.82 \\
$\mathrm{M}_{1}$ & 1.10 & 1.06 & 1.14 \\
$\mathrm{M}_{6}$ & 1.56 & 1.49 & 1.63 \\
$\mathrm{M}_{7}$ & 1.37 & 1.32 & 1.45 \\
$\mathrm{~T}_{0}$ & 0.067 & 0.077 & 0.083 \\
$\mathrm{~T}_{1}$ & 0.036 & 0.033 & 0.036 \\
$\mathrm{~T}_{2}$ & 0.119 & 0.111 & 0.119 \\
\hline
\end{tabular}

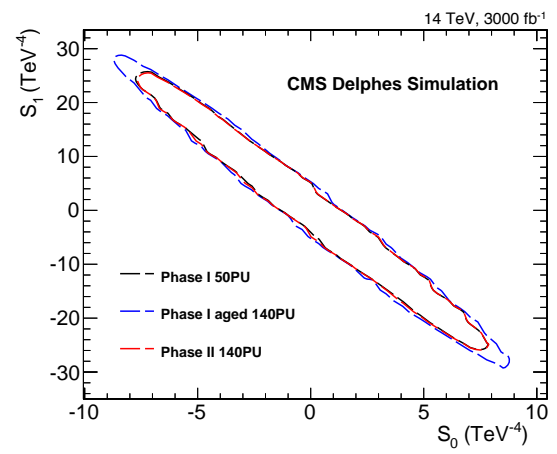

Figure 4: Expected 95\% CL limits on the coefficients for the BSM higher-order (dimension eight) operators in the EFT Lagrangian (left) and 2D contour for the $S_{0}$ and $S_{1}$ parameters (right). 


\section{References}

[1] V. Khachatryan et al. [CMS Collaboration], "Study of vector boson scattering and search for new physics in events with two same-sign leptons and two jets", Phys. Rev. Lett. 114 (2015) 5, 051801 [arXiv:1410.6315 [hep-ex]].

[2] G. Aad et al. [ATLAS Collaboration], "Evidence for Electroweak Production of $W^{ \pm} W^{ \pm} j j$ in $p p$ Collisions at $\sqrt{s}=8 \mathrm{TeV}$ with the ATLAS Detector”, Phys. Rev. Lett. 113, no. 14, 141803 (2014) [arXiv:1405.6241 [hep-ex]].

[3] B. W. Lee, C. Quigg and H. B. Thacker, "The Strength of Weak Interactions at Very High-Energies and the Higgs Boson Mass", Phys. Rev. Lett. 38, 883 (1977).

[4] CMS Collaboration, "Technical Proposal for the Phase-II Upgrade of the CMS Detector", CERN, CERN-LHCC-2015-010. LHCC-P-008.

[5] O. J. P. Eboli, M. C. Gonzalez-Garcia and J. K. Mizukoshi, " $p p \rightarrow j j e^{ \pm} \mu^{ \pm} v v$ and $j j e^{ \pm} \mu^{ \pm} v v$ at $\mathrm{O}\left(\alpha_{e w}^{6}\right)$ and $\mathrm{O}\left(\alpha_{e w}^{4} \alpha_{s}^{2}\right)$ for the study of the quartic electroweak gauge boson vertex at CERN LHC", Phys. Rev. D 74, 073005 (2006) [hep-ph/0606118].

[6] A. Ballestrero, A. Belhouari, G. Bevilacqua, V. Kashkan and E. Maina, "PHANTOM: A Monte Carlo event generator for six parton final states at high energy colliders", Comput. Phys. Commun. 180, 401 (2009) [arXiv:0801.3359 [hep-ph]].

[7] J. Alwall, M. Herquet, F. Maltoni, O. Mattelaer and T. Stelzer, "MadGraph 5 : Going Beyond", JHEP 1106, 128 (2011) [arXiv:1106.0522 [hep-ph]].

[8] J. de Favereau et al. [DELPHES 3 Collaboration], "DELPHES 3, A modular framework for fast simulation of a generic collider experiment”, JHEP 1402, 057 (2014) [arXiv:1307.6346 [hep-ex]].

[9] C. Degrande, N. Greiner, W. Kilian, O. Mattelaer, H. Mebane, T. Stelzer, S. Willenbrock and C. Zhang, "Effective Field Theory: A Modern Approach to Anomalous Couplings", Annals Phys. 335, 21 (2013) [arXiv:1205.4231 [hep-ph]].

[10] CMS Collaboration, "Performance of b tagging at $\sqrt{s}=8 \mathrm{TeV}$ in multijet, $t \bar{t}$ and boosted topology events”, CMS-PAS-BTV-13-001.

[11] K. Doroba, J. Kalinowski, J. Kuczmarski, S. Pokorski, J. Rosiek, M. Szleper and S. Tkaczyk, "The $W_{L} W_{L}$ Scattering at the LHC: Improving the Selection Criteria”, Phys. Rev. D 86, 036011 (2012) [arXiv:1201.2768 [hep-ph]]. 